\title{
Coupling dominant surface submodels and complex physical process Computational Fluid Dynamics
}

\section{Greg Perkins*}

(Received 8 August 2003; revised 27 January 2004)

\begin{abstract}
Many processes in the mineral, power and chemical industries involve simulating combined heat and mass transfer with heterogeneous chemical reactions. While numerical methods and codes for solving heterogeneous reactions involving particles and/or droplets are widely available, numerical methods and codes for solving strongly coupled surface submodels are far less widespread and far less robust. In this paper, a solver for arbitrary heterogeneous chemical reactions is developed and linked to a general purpose CFD code, to solve coupled heat and mass transfer problems with dominant surface reactions. The
\end{abstract}

*School of Materials Science and Engineering, University of New South Wales, Sydney, Australia. Present Address: Shell International Chemicals BV, Amsterdam, The NetherLands. mailto:greg.perkins@shell.com

See http://anziamj.austms.org.au/V45/CTAC2003/Perk/home.html for this article, (c) Austral. Mathematical Soc. 2004. Published August 8, 2004. ISSN 1446-8735 
chemical reaction solver uses the Newton-Raphson technique and LUdecomposition to solve the nonlinear species mass balance equations at the reacting surface. Issues with integrating the chemical reaction solver with the CFD code are discussed and the model is then applied to the simulation of underground coal gasification.

\section{Contents}

1 Introduction

C818

2 Surface reaction submodel

C819

2.1 Mass balance . . . . . . . . . . . . . . . . . . C820

2.2 Energy balance . . . . . . . . . . . . . . . . . . . . C C821

2.3 Numerical method . . . . . . . . . . . . . . . . . C822

2.4 Coupling with CFD . . . . . . . . . . . . . . . . C822

3 Application to underground coal gasification $\quad \mathbf{C 8 2 3}$ 3.1 Description of CFD model . . . . . . . . . . . . . C824

3.2 Results . . . . . . . . . . . . . . . . . C 825

4 Conclusions

C829

References

C829

\section{Introduction}

While surface reactions are important in a wide range of industrial processes, including chemical vapour deposition and underground coal gasification [5], they have received far less attention in the literature than reactions involving particles and droplets. 
In this paper a submodel for solving heterogeneous surface reactions is developed and linked to a general purpose CFD code. The submodel uses a Newton-Raphson iterative technique together with $L U$-decomposition to solve the nonlinear species balance equations at the reacting surface. The solver has been found to be efficient, robust and capable of handling numerically stiff chemical systems.

The combined surface reaction submodel and CFD solver has been successfully applied to simulate the tightly coupled physical processes of heat, mass and momentum transfer with chemical reactions in an underground coal gasification (UCG) system. Dry gas compositions from the model are compared with field trial results and insights into the process are discussed.

\section{Surface reaction submodel}

Throughout this work, a generalised treatment of a chemical reaction is assumed, with the $k$ th reaction written as

$$
\sum_{i=1}^{N} \nu_{i, k}^{\prime} \mathcal{M}_{i} \underset{k_{b, k}}{\stackrel{k_{f, k}}{\rightleftharpoons}} \sum_{i=1}^{N} \nu_{i, k}^{\prime \prime} \mathcal{M}_{i}
$$

The molar rate of chemical reaction $k$ is

$$
R_{k}=k_{f, k} \prod_{j=1}^{N}\left[C_{j}\right]^{\eta_{j, k}^{\prime}}-k_{b, k} \prod_{j=1}^{N}\left[C_{j}\right]^{\eta_{j, k}^{\prime \prime}}
$$

and the rate of creation/destruction of the $i$ th species in reaction $k$ is calculated with

$$
\hat{R}_{i, k}=\left(\nu_{i, k}^{\prime \prime}-\nu_{i, k}^{\prime}\right)\left(k_{f, k} \prod_{j=1}^{N}\left[C_{j}\right]^{\eta_{j, k}^{\prime}}-k_{b, k} \prod_{j=1}^{N}\left[C_{j}\right]^{\eta_{j, k}^{\prime \prime}}\right) .
$$


In Equation (3) the forward rate, $k_{f, k}$ is assumed to have the Arrhenius form $k_{f, k}=A_{k} T^{\alpha_{k}} \exp \left(-E_{k} / R T\right)$ and the backward rate is calculated using thermodynamic equilibrium: $k_{b, k}=k_{f, k} / K_{C, k}$.

\subsection{Mass balance}

Chemical reaction at a surface involves a balance between the diffusion of reactants to the surface, and advection of products away from the surface plus a consideration of the kinetics of the reaction. Under pseudo-steadystate conditions a species balance at the surface yields

$$
-\rho_{g} V Y_{s}^{i}-\rho_{g} D_{\mathrm{eff}, i} \frac{\partial Y^{i}}{\partial n}+\dot{\omega}_{s}^{i}=0, \quad i=1, \ldots, N_{g} .
$$

By discretising the species gradient term and substituting an overall masstransfer co-efficient as $h_{m, i}=D_{\mathrm{eff}, i} / \Delta n$ and recognising that the total mass flux through the surface is the sum of the species mass fluxes, that is: $\rho_{g} V=$ $\sum_{j=1}^{N_{g}} \dot{\omega}_{s}^{i}$, Equation (4) is rearranged to give

$$
-\sum_{j=1}^{N_{g}} \dot{\omega}_{s}^{j} Y_{s}^{i}-\rho_{g} h_{m, i}\left(Y_{s}^{i}-Y_{g}^{i}\right)+\dot{\omega}_{s}^{i}=0, \quad i=1, \ldots, N_{g}-1 .
$$

Since the species mass fractions must sum to one, only $N_{g}-1$ nonlinear equations of the form (5) are required, with the last species having the value: $Y_{s}^{N_{g}}=1-\sum_{i=1}^{N_{g}-1} Y_{s}^{i}$. The $\dot{\omega}_{s}^{i}$ term represents the net mass source of species $i$ at the surface $\left(\mathrm{kg} / \mathrm{m}^{2}-\mathrm{s}\right)$ and is calculated using

$$
\dot{\omega}_{s}^{i}=W_{i} \sum_{k=1}^{N_{R}} \hat{R}_{i, k}+\dot{\varphi}_{s}^{i},
$$

where $W_{i}$ is the molecular weight of species $i$. See in Equation (6) that the net mass source of species $i$ is calculated as the sum of the sources from 
each reaction that the species participates in, plus an additional term $\dot{\varphi}_{s}^{i}$. The source term $\dot{\varphi}_{s}^{i}$ accounts for thermal decomposition and moisture evaporation that occurs behind the surface reaction front due to heat transfer into the material. The rates are determined by making use of the ultimate analysis of the material:

$$
\begin{aligned}
& \dot{\varphi}_{s}^{i}=\dot{\varphi}_{s}^{\mathrm{VM}, i}+\delta_{i, H_{2} O} \dot{\varphi}_{s}^{\mathrm{H}_{2} O}, \\
& \dot{\varphi}_{s}^{\mathrm{VM}, i}=\dot{\omega}_{s}^{\text {char }} \cdot\left(\frac{Y_{\mathrm{VM}, i}}{Y_{C}+Y_{A}}\right), \\
& \dot{\varphi}_{s}^{H_{2} O}=\dot{\omega}_{s}^{\text {char }} \cdot\left(\frac{Y_{W}}{Y_{C}+Y_{A}}\right)+\dot{\varphi}_{s}^{\text {water }},
\end{aligned}
$$

where $Y_{C}, Y_{W}$ and $Y_{A}$ represent the fraction of fixed carbon, moisture and ash in the material, $Y_{\mathrm{VM}, i}$ is the fraction of the $i$ th gas species in the volatile matter and $\dot{\varphi}_{s}^{\text {water }}$ is a mass flux of water to the surface from the surroundings due to hydrostatic pressure.

\subsection{Energy balance}

Once the mass balance is known the energy balance can be calculated. The net heat flux into the material is calculated from

$$
Q_{s}=\sum_{k=1}^{N_{R}} R_{k} \Delta H_{k}+\dot{\varphi}_{s}^{\mathrm{H}_{2} \mathrm{O}} \Delta H_{\mathrm{vap}}+\dot{\varphi}_{s}^{\text {char }} C p \cdot\left(T_{s}-T_{\infty}\right),
$$

where the first term accounts for the heat of reaction of each surface reaction, the second term accounts for evaporation of moisture and the third term accounts for heating of the material from the ambient temperature $\left(T_{\infty}\right)$ to the surface temperature $\left(T_{s}\right)$. 


\subsection{Numerical method}

The iterative Newton-Raphson technique [1] solves Equation (5). The method is robust and has the advantage that numerically stiff chemical systems are solved efficiently. The function $F_{i}$ is formed from Equation (5) as

$$
F_{i}=-\sum_{j=1}^{N_{g}} \dot{\omega}_{s}^{j} Y_{s}^{i}-\rho_{g} h_{m, i}\left(Y_{s}^{i}-Y_{g}^{i}\right)+\dot{\omega}_{s}^{i}
$$

and is expanded in a Taylor series in the neighbourhood of the current solution. By neglecting terms greater than first order, a linear system of equations is obtained for the surface mass fraction corrections $\delta Y_{s}^{i}$ that move the set of balance equations such that $F_{i} \rightarrow 0$,

$$
F_{i}=-J_{i, k} \delta Y_{s}^{i}
$$

The linear system (12) is solved at each iteration using the $L U$-decomposition method [1]. The surface mass fractions are updated with $Y_{s}^{i, n+1}=Y_{s}^{i, n}+\delta Y_{s}^{i}$ and the procedure is repeated until specified error tolerances on the solution are met. The entries in the Jacobian matrix

$$
J_{i, k}=\frac{\partial F_{i}}{\partial Y_{s}^{k}}=-\sum_{j=1}^{N_{g}}\left(\frac{\partial \dot{\omega}_{s}^{j}}{\partial Y_{s}^{k}} Y_{s}^{i}+\delta_{i, k} \dot{\omega}_{s}^{j}\right)-\delta_{i, k} \rho_{g} h_{m, i}+\frac{\partial \dot{\omega}_{s}^{i}}{\partial Y_{s}^{k}},
$$

where the derivative term $\partial \dot{\omega}_{s}^{j} / \partial Y_{s}^{k}$ is further expanded using Equations (6) and (3).

\subsection{Coupling with CFD}

The surface reaction submodel is written in $\mathrm{C}$ and the definitions of the individual species, material properties, thermodynamic data and reaction rate expressions are read from a data file. The submodel is coupled to the 
general purpose CFD code FLUENT through user defined functions [2]. The fluxes of mass, species and momentum which are the outputs of the surface reaction submodel, are added to the appropriate conservation equations in the near-wall cells as volumetric source terms by applying the divergence theorem. The net heat flux calculated using Equation (10) provides the wall boundary condition for the energy equation.

During testing of the surface reaction submodel coupled to the CFD solver, it was found that direct under-relaxation of the surface reaction rates was helpful in improving numerical stability and the convergence behaviour of the complete model. Under-relaxation is applied to the surface-reaction rates according to

$$
R_{k}^{n+1}=R_{k}^{n}+\alpha_{R}\left(R_{k}^{n+1}-R_{k}^{n}\right),
$$

where a value of $0.3<\alpha_{R}<0.5$ was found to be optimal for the problems described in this paper.

\section{Application to underground coal gasification}

To demonstrate the capabilities of the model, an underground coal gasification (UCG) system of the borehole type used during the Newman Spinney P5 field trial is considered [6]. In this system air was injected into a borehole drilled in the coal seam where it reacted on the surface of the coal to form a product gas of carbon monoxide, hydrogen and methane together with nitrogen and steam. The geometry of the gasifier and operating conditions are shown in Table 1. 
TABLE 1: Operating conditions for the borehole UCG model

\begin{tabular}{lcc}
\hline Parameter & Value & Unit \\
\hline Borehole length & 140 & $\mathrm{~m}$ \\
Borehole diameter & 0.2 & $\mathrm{~m}$ \\
Pressure & 200 & $\mathrm{kPa}$ \\
Inlet mass flow & 2.5 & $\mathrm{~kg} / \mathrm{s}$ \\
Inlet gas & air & \\
\hline
\end{tabular}

\subsection{Description of CFD model}

The FLUENT CFD model of the borehole uses an axisymmetric coordinate system and solves for the conservation of mass, species, energy, momentum and uses the standard $k-\varepsilon$ turbulence model. The $\mathrm{P} 1$ radiation model is used to account for radiation heat transfer. Due to space restrictions a full description of the solved equations is not given here, but is to be found in the FLUENT manual [2].

The conservation equations are solved with a segregated multigrid finite volume solver based on the SIMPLE algorithm. A second order upwind scheme has been used to discretise the convective terms of the conservation equations.

Thermodynamic properties of the gas phase are calculated using standard techniques and account for the dependencies on temperature and composition. Coal ultimate and proximate analyses are taken from Gibb [6]. Chemical reactions occurring in the system are given in Table 2. Coal char is considered to be pure carbon. Reactions R1-R3 are heterogeneous and are calculated using the surface reaction submodel described in Section 2. The homogeneous combustion reactions $\mathrm{R} 4$ and $\mathrm{R} 5$ are calculated by a finiterate/eddy-breakup model [3]. The water-gas-shift reaction, $\mathrm{R} 6$ is modelled as an equilibrium reaction. 
TABLE 2: Important chemical reactions during underground coal gasification (kinetic data is taken from the indicated references)

\begin{tabular}{lll}
\hline Reaction & Stoichiometry & Ref. \\
\hline R1 & $\mathrm{C}+\frac{1}{2} \mathrm{O}_{2} \rightarrow \mathrm{CO}$ & {$[7]$} \\
R2 & $\mathrm{C}+\mathrm{H}_{2} \mathrm{O} \rightarrow \mathrm{CO}+\mathrm{H}_{2}$ & {$[7]$} \\
R3 & $\mathrm{C}+\mathrm{CO}_{2} \rightarrow 2 \mathrm{CO}$ & {$[7]$} \\
R4 & $\mathrm{CO}+\frac{1}{2} \mathrm{O}_{2} \rightarrow \mathrm{CO}_{2}$ & {$[8]$} \\
R5 & $\mathrm{H}_{2}+\frac{1}{2} \mathrm{O}_{2} \rightarrow \mathrm{H}_{2} \mathrm{O}$ & {$[8]$} \\
R6 & $\mathrm{H}_{2} \mathrm{O}+\mathrm{CO} \rightleftharpoons \mathrm{H}_{2}+\mathrm{CO}_{2}$ & {$[4]$} \\
\hline
\end{tabular}

TABle 3: Comparison of dry gas compositions (mole \%)

\begin{tabular}{lrr}
\hline Species & Field Data & Model \\
\hline $\mathrm{CO}$ & 8.0 & 8.8 \\
$\mathrm{CO}_{2}$ & 15.0 & 14.1 \\
$\mathrm{H}_{2}$ & 9.4 & 10.5 \\
$\mathrm{CH}_{4}$ & 0.8 & 1.3 \\
$\mathrm{~N}_{2}$ & 66.5 & 65.3 \\
\hline
\end{tabular}

\subsection{Results}

Since the water influx rate during the trial period is uncertain, it has been adjusted so as to obtain a good match between the reported and predicted dry gas compositions. A value of $\dot{\varphi}_{s}^{\text {water }}=0.01 \mathrm{~kg} / \mathrm{m}^{2}$ was found to give a reasonable match and the comparison of dry gas compositions is given in Table 3.

Profiles of gas and wall temperatures, gas species concentrations and surface reaction rates calculated by the model are shown in Figures 1,2 and 3, 


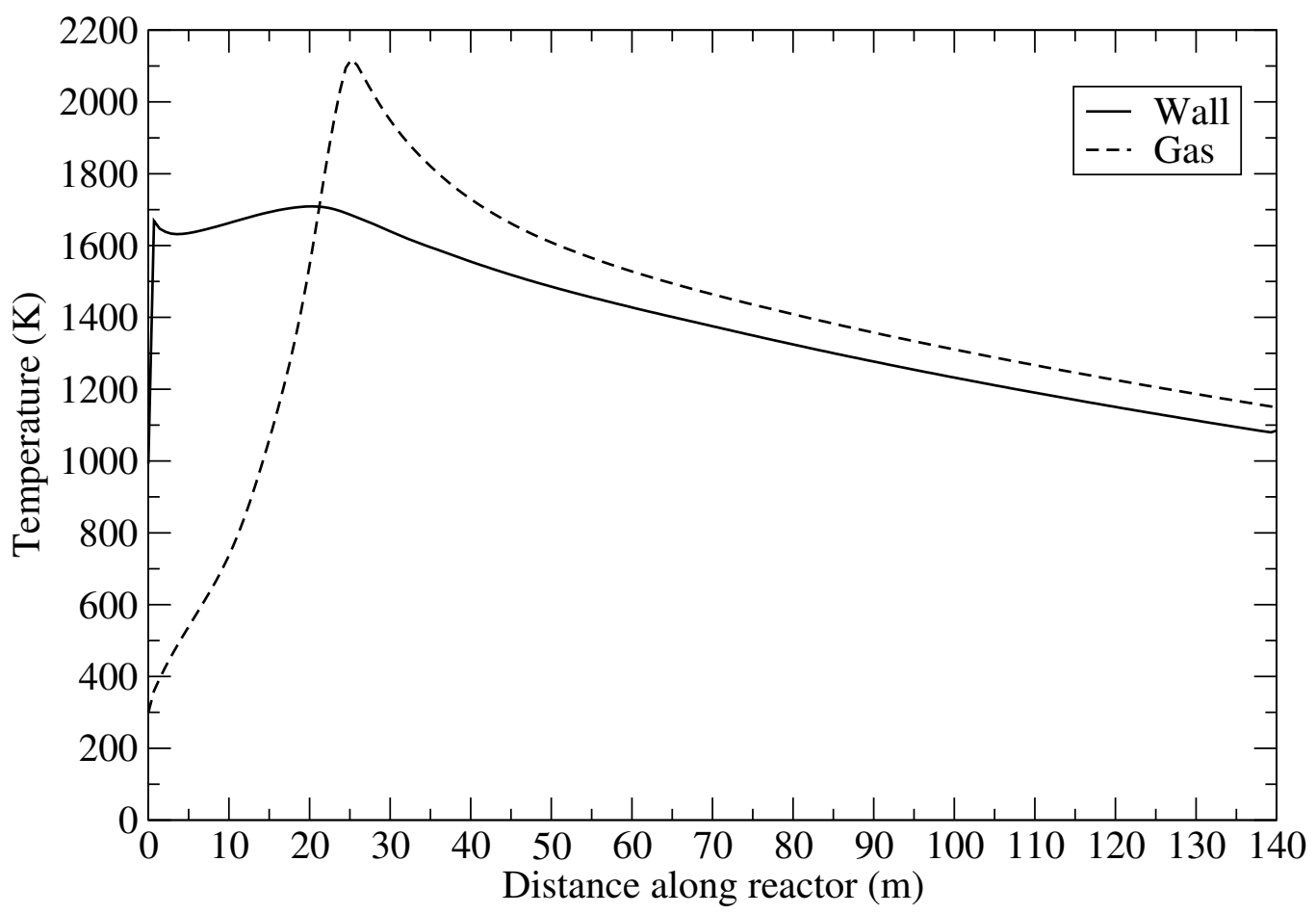

Figure 1: Profile of wall and gas temperature (K) 


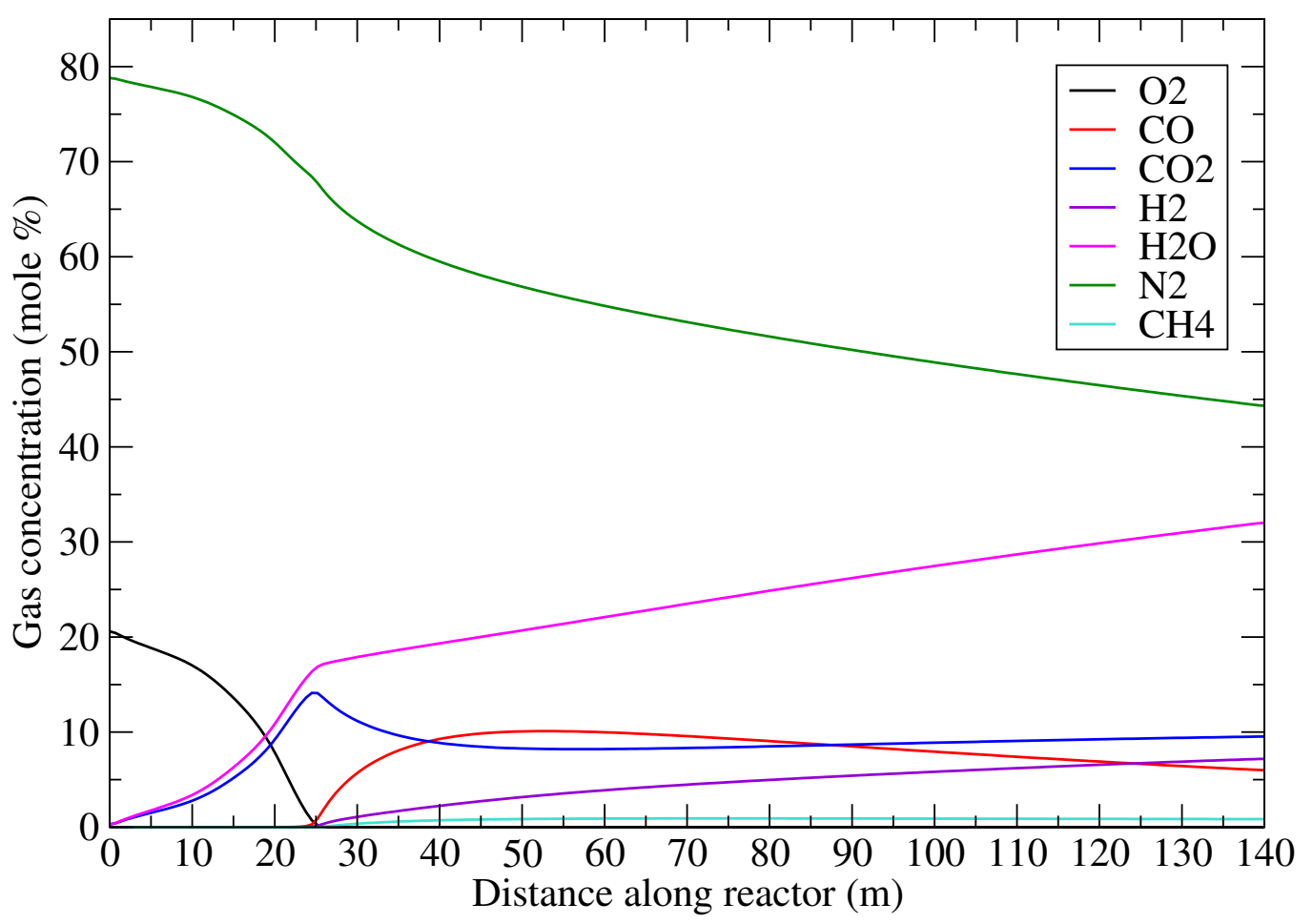

Figure 2: Profile of gas species concentration (mole \%) 


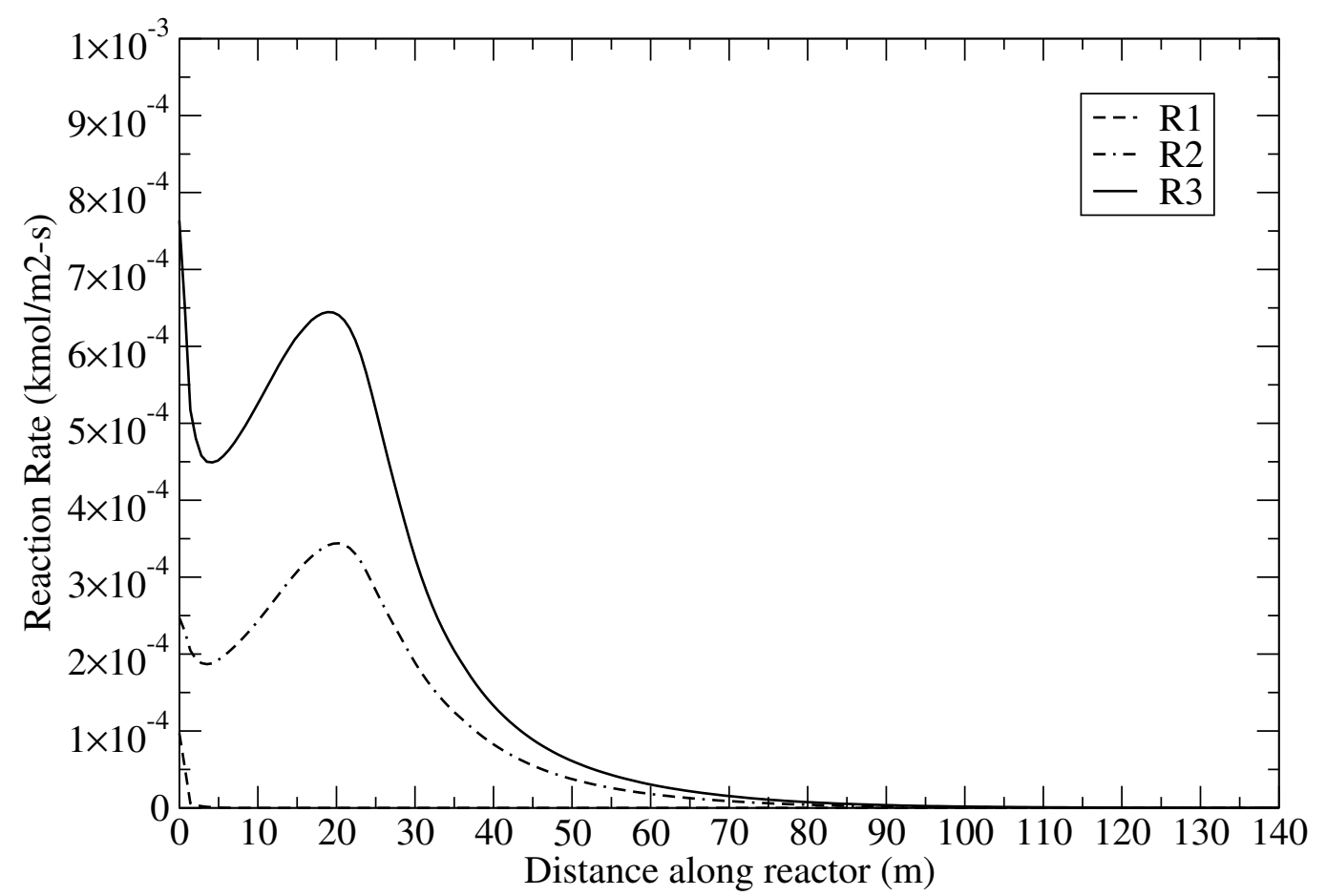

Figure 3: Profile of surface reaction rates $(\mathrm{kmol} / \mathrm{m} 2-\mathrm{s})$ 
respectively. The peak temperature at $\sim 25 \mathrm{~m}$ coincides with depletion of oxygen from the gas phase and marks the beginning of the reduction zone, in which the combustible gases $\mathrm{CO}, \mathrm{H}_{2}$ and $\mathrm{CH}_{4}$ are produced. The linear increase in steam concentration is primarily a result of the water influx source term $\dot{\varphi}_{s}^{\text {water }}$. Profiles of the surface reaction rates show that the highest coal consumption rates occur in the high temperature region and that the reactions $\mathrm{R} 2$ and $\mathrm{R} 3$ have similar reaction rates. While the char combustion reaction $\mathrm{R} 1$ plays an insignificant role in forming products, it provides a critical ignition mechanism within the model.

\section{Conclusions}

In this paper a submodel for surface reactions has been developed and linked through user code to a general CFD code. The use of a Newton-Raphson technique in combination with $L U$-decomposition has proven to be robust and efficient. It has been found that under-relaxation of the chemical reaction rates between iterations improves numerical stability and convergence behaviour. Application of the combined surface reaction and CFD model to the problem of underground coal gasification has been successful. The model is useful for gaining insights into the behaviour of the underground coal gasification process and because of its general nature can be easily applied to other systems in which surface reactions are important.

\section{References}

[1] Numerical Recipes in C. Cambridge University Press, 1992. C822

[2] FLUENT 6.0 Users Guide. Fluent Inc., Lebanon, NH, December 2001. $\mathrm{C} 823, \mathrm{C} 824$ 
[3] Jan Roar Bakke and Bjorn H. Hjertager. The effect of explosion venting in empty vessels. Intl. J. Num. Meth. Eng, 24:129-140, 1987. C824

[4] Colomba Di Blasi. Dynamic behaviour of stratified downdraft gasifiers. Chem. Eng. Sci., 55:2931-2944, 2000. C825

[5] B. Dinsmoor, J. M. Galland, and T. F. Edgar. The modeling of cavity formation during underground coal gasification. J. Pet. Tech., pages 695-704, May 1978. C818

[6] Alexander Gibb. The Underground Gasification of Coal. Sir Isaac Pitman \& Sons Ltd, London, 1964. C823, C824

[7] Michael L. Hobbs, Predrag T. Radulovic, and L. Douglas Smoot. Modeling fixed-bed coal gasifiers. A. I. C. H. E. J., 38(5):681-701, 1992. C825

[8] W. P. Jones and R. P. Lindstedt. Global reaction schemes for hydrocarbon combustion. Comb. \& Flame, 73:233-249, 1988. C825 